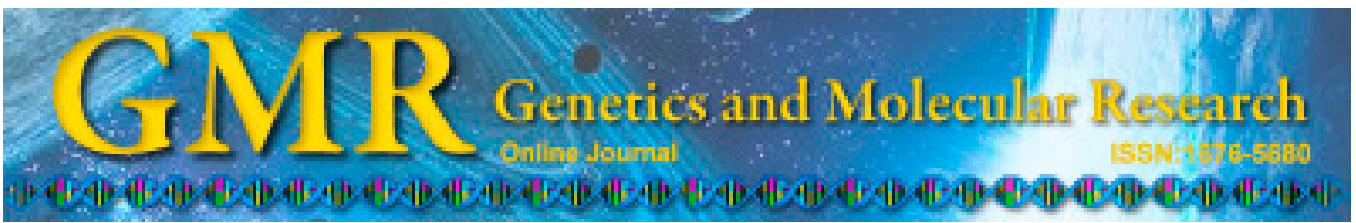

\title{
Characteristic element of matrix attachment region mediates vector attachment and enhances nerve growth factor expression in Chinese hamster ovary cells
}

\author{
X.Y. Wang, J.H. Zhang, Q.L. Sun, Z.Y. Yao, B.G. Deng, W.Y. Guo, \\ L. Wang, W.H. Dong, F. Wang, C.P. Zhao and T.Y. Wang \\ Department of Biochemistry and Molecular Biology, \\ Xinxiang Medical University, Xinxiang, Henan Province, China \\ Corresponding author: T.Y. Wang \\ E-mail: 862001455@qq.com
}

Genet. Mol. Res. 14 (3): 9191-9199 (2015)

Received February 3, 2015

Accepted May 14, 2015

Published August 7, 2015

DOI http://dx.doi.org/10.4238/2015.August.7.29

\begin{abstract}
Preliminary studies have suggested that a characteristic element of the matrix attachment region (MAR) in human interferon- $\beta$ mediates the adhesion of vectors to Chinese hamster ovary (CHO) cells. In this study, we investigated if vector adhesion increased nerve growth factor (NGF) expression in $\mathrm{CHO}$ cells. The MAR characteristic element sequence of human interferon- $\beta$ was inserted into the multiple-cloning site of the $\mathrm{pEGFP}-\mathrm{C} 1$ vector. The target NGF gene was inserted upstream of the MAR characteristic element sequence to construct the MAR/ NGF expression vector. The recombinant plasmid was transfected into $\mathrm{CHO}$ cells and stable monoclonal cells were selected using G418. NGF mRNA and protein expression was detected by reverse transcriptasepolymerase chain reaction and enzyme-linked immunosorbent assay, respectively. Plasmid reduction experiments were used to determine the state of transfected plasmid in mammalian cells. The insertion of MAR
\end{abstract}


into the vector increased NGF expression levels in CHO cells (1.93fold) compared to the control. The recombinant plasmid expressing the MAR sequence was digested into a linear space vector. The inserted MAR and NGF sequences were consistent with those inserted into the plasmid before recombination. Therefore, we concluded that the MAR characteristic element mediates vector adhesion to $\mathrm{CHO}$ cells and enhances the stability and efficiency of the target gene expression.

Key words: Matrix attachment region (MAR); Characteristic element; Attached vector; Transgene expression; Chinese hamster ovary cell; Nerve growth factor; $\beta$-interferon MAR

\section{INTRODUCTION}

Gene therapy is a novel field of research in the medical and biological sciences, and has gradually developed in conjunction with the recent advances in recombinant DNA technology. The application of vectors is a crucial step in gene therapy. There are two types of vectors: integrated and non-integrated vectors. Integrated vectors facilitate the insertion of mutations, and may be responsible for diseases arising as a result of the position of the inserted gene (Buceta et al., 2011; Ley et al., 2013). Ideal non-integrated vectors present no potential risk in gene therapy, are stable through mitosis, and express high levels of the transgene (Girod et al., 2007). Matrix attachment region (MAR) (200-2000 bp), or the scaffold attachment region, is a DNA sequence rich in AT base pairs $(>60 \%)$ attached to the matrix after enzymatic digestion. The MAR provides a cis-acting element to the attached vector, avoids epigenetic gene silencing, and promotes the stability of mitotic chromosomes (Kamimura et al., 2011; Harraghy et al., 2011, 2012). The MAR sequence is a special DNA sequence that binds to the nuclear matrix. It is located in the bilateral non-coding regions of genes. MAR contains some characteristic sequence components, including a T-box (TTTTATTTTT), an A-box (AATAAAAA/CAA), an autonomous replicating DNA sequence, and fruit fly topoisomerase II recognition sites (Ehrhardt et al., 2008; Mucller and Flotte, 2008). The MAR also mediates the attachment of vectors to host cells (Chiaretti et al., 2008; Zheng et al., 2010; Calado et al., 2014).

Nerve growth factor (NGF) is an important bioactive molecule in the nervous system. NGF plays an important role in maintaining the growth and development of the nervous system, and participates in the regeneration and repair of damaged nerves. Therefore, exogenous NGF has been increasingly applied to the treatment of cerebral injuries in clinical settings. However, NGF cannot easily penetrate the blood brain barrier because of its large molecular weight. Additionally, the intravenous administration of $N G F$ does not allow for optimum efficiency. In addition to its high cost, these factors limit the application of NGF to the treatment of disorders and defects in the central nervous system (Haase et al., 2010; Liu et al., 2013). Gene transfection is a novel tool that can be used in the application of multipleneurotrophic factors. Preliminary studies have shown that the MAR characteristic element in human interferon- $\beta$ can mediate the adhesion of vectors to Chinese hamster ovary $(\mathrm{CHO})$ cells. However, its possible effect on enhancing the transgene expression remains to be determined. 


\section{MATERIAL AND METHODS}

\section{Vector construction}

MAR characteristic motifs, such as the AT sequences in interferon- $\beta$ ( $2200 \mathrm{bp}$, GenBank: M83137.1), were cut and spliced to short MAR characteristic sequences (367 bp). MAR was amplified by polymerase chain reaction (PCR), and directed cloning was performed using the KpnI and BamHI enzyme restriction sites generated in the 5'-terminus of the primers. The PCR conditions were as follows: $95^{\circ} \mathrm{C}$ for $3 \mathrm{~min}, 94^{\circ} \mathrm{C}$ for $40 \mathrm{~s}, 60-56^{\circ} \mathrm{C}$ for $30 \mathrm{~s}$, and $72^{\circ} \mathrm{C}$ for $40 \mathrm{~s}$. Each annealing temperature was used for 4 cycles. Finally, the PCR product was amplified using an annealing temperature of $55^{\circ} \mathrm{C}$ for 20 cycles. A final extension step was performed at $72^{\circ} \mathrm{C}$ for $3 \mathrm{~min}$. The T-vector (TaKaRa Bio, Inc., Dalian, China), connected to the MAR fragment and the pEGFP-C1 vector, was digested using the corresponding restriction enzymes. The intermediate vector, pEGFP-C1-MAR, was then constructed. Specific NGF primers were designed for the $N G F$ gene (GenBank: AF150960.1). DNA from human peripheral blood was used as the template to amplify NGF. HindIII and KpnI restriction sites were introduced. pEGFP-C1 and pEGFP-C1-MAR vectors were amplified by PCR, and the NGF was directionally inserted upstream of MAR using the multiple-cloning site of the pEGFP-C1 vector. The pEGFP-C1-NGF and pEGFP-C1-MAR-NGF were then constructed, and used for further digestion and sequencing to verify the correctness of the sequences (Figure 1).

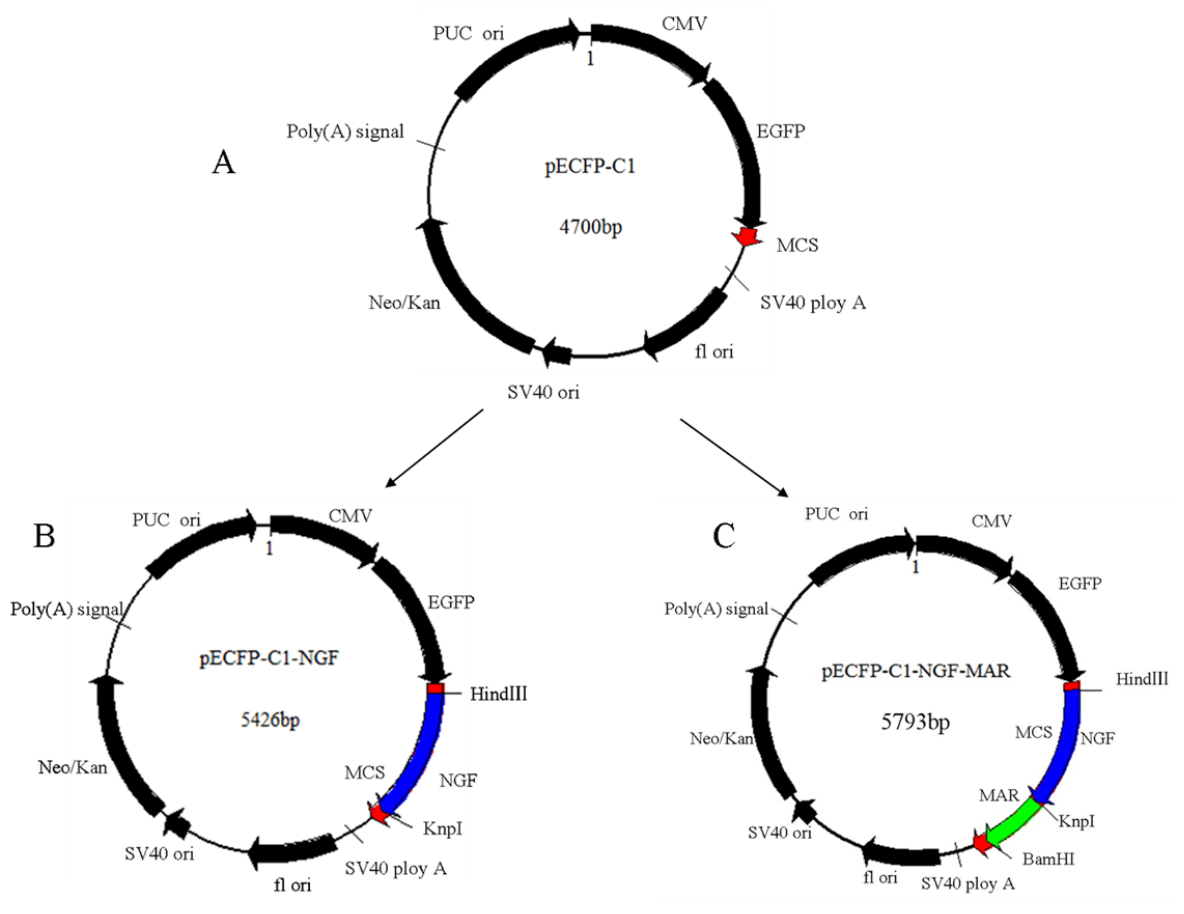

Figure 1. Plasmid vectors used in this study. A.Vector pEGFP-C1. B. Expression vector pEGFP-C1-NGF containing NGF at the MCS site. C. Expression vector pEGFP-C1-NGF-MAR containing MAR and NGF. 


\section{Cell culture and transfection}

CHO cells (Institute of Laboratory Animal Sciences, Beijing, China) were cultured in Dulbecco's modified Eagle's medium (DMEM; Gibco, Grand Island, NY, USA), supplemented with $10 \%$ inactivated fetal calf serum (Gibco) on a 6 -well plate $\left(3 \times 10^{6}\right.$ cells/ well) at $37^{\circ} \mathrm{C}$ and $5 \% \mathrm{CO}_{2}$. Three transfection groups were prepared: the pEGFP-C1-NGF vector transfection group, $\mathrm{pEGFP-C1-MAR-NGF} \mathrm{vector} \mathrm{transfection} \mathrm{group,} \mathrm{and} \mathrm{pEGFP-}$ $\mathrm{C} 1$ negative control group. The transfection was performed using the Sunma-sofast gene transfection reagent kit (Sunma Biotechnology Co., Ltd., Xiamen, China) according to the manufacturer protocols. G418 (Calbiochem, La Jolla, CA, USA; final concentration: 800 $\mu \mathrm{g} / \mathrm{mL}$ ) was used $24 \mathrm{~h}$ after transfection to select the transfected cells for 2 weeks. The culture medium was changed every 2-3 days. Stable transformed cell colonies were trypsinized $(0.25 \%$ trypsin). Transfected cells in each group were selected by the dilution method, in order to obtain the monoclonal cell lines. These cells were then split onto a 96-well culture plate, and transferred to culture flasks after 7 days. The cells were analyzed at a cell density of up to $80-90 \%$.

\section{Reverse transcriptase-PCR (RT-PCR)}

The collected cells were adjusted to the same concentration in each group $\left(1 \times 10^{6} / \mathrm{mL}\right)$, and the same amount of cells was collected (via centrifugation) for each group. The total RNA was extracted from these cells using the Total RNA Isolation kit (TaKaRa Bio Inc.). RNA was reverse transcribed to cDNA using the RT-PCR Kit (TaKaRa Bio Inc.). The primers designed based on the $N G F$ gene were P1: 5'-ATGTCCATGTTGTTCTACACTCT-3' and P2: 5'-TCAGGCTCTTCTCACAGCCTTCCT-3'. The PCR product was 726 bp long. The GAPDH control primers were P3: 5'-CACATATTCTGGAGGAGCCTCC-3' and P4: 5'-ACGGTGCCATGGAATTTGCCAT-3'. The PCR product was 295 bp long. These primers were synthesized by Sangon Biotechnology Development Co., Ltd. (Shanghai, China). The target and internal reference genes were amplified using the reverse transcribed cDNA. The PCR conditions were as follows: initial denaturation at $95^{\circ} \mathrm{C}$ for $5 \mathrm{~min} ; 30$ cycles of $94^{\circ} \mathrm{C}$ for $40 \mathrm{~s}, 55^{\circ} \mathrm{C}$ for $40 \mathrm{~s}$, and $72^{\circ} \mathrm{C}$ for $30 \mathrm{~s}$; and a final extension at $72^{\circ} \mathrm{C}$ for $5 \mathrm{~min}$. The amplified PCR products were separated by electrophoresis, using a $2 \%$ agarose gel. The target bands were imaged using a UV analyzer, and the Bander leader 3.0 gel image processing software was used to analyze the image. The fold-change of the target gene over the GAPDH gene reflected the relative expression level of target mRNA.

\section{NGF concentration analysis}

The DMEM culture was replaced when a cell density of $80-90 \%$ was attained. The cell culture supernatant was collected. The cells were counted after $24 \mathrm{~h}$. The NGF secretory protein concentration in the cell culture supernatant was measured using NGF enzyme-linked immunosorbent assay kit (DingGuo Biological Technology Co., Ltd., Beijing, China). The experiment was repeated three times. 


\section{Plasmid reduction}

The attached vectors were isolated from the CHO cells using the Hirt (1967) cracking method, and subsequently transformed. Positive transformants were selected and cultured in tubes with the LK liquid medium overnight at $37^{\circ} \mathrm{C}$ on a shaking table. The plasmids were isolated from the cells using the plasmid isolation kit (Solarbio, Beijing, China) according to the manufacturer protocols. The plasmids were digested with KpnI and BamHI or HindIII and $K p n I$, and subsequently digested with $K p n I$.

\section{Statistical analysis}

All data were statistically analyzed using the SPSS v.18.0 software platform (SPSS Inc., Chicago, IL, USA). All data are reported as means \pm standard deviation. The differences among different groups were estimated by ANOVA. P values $<0.05$ were considered to be statistically significant.

\section{RESULTS}

\section{Vector construction}

The constructed vectors were digested using the different enzymes. Agarose gel electrophoresis indicated the presence of a 726-bp fragment in pEGFP-C1-NGF, when digested with HindIII and KpnI; however, a linear DNA fragment was obtained after digestion with $K p n I$. These findings demonstrated the successful construction of the pEGFP-C1-NGF vector. The pEGFP-C1-MAR-NGF vector was digested using KpnI and BamHI, as well as HindIII and $K p n I$. Two fragments were obtained (367 and 726 bp, respectively). A linear DNA fragment was upon digestion with $K p n I$. The results were consistent with the expected fragment sizes. Sequencing results also demonstrated the successful construction of the pEGFP-C1NGF and pEGFP-C1-MAR-NGF expression vectors.

\section{RT-PCR}

A 726-kb band, specific for the $N G F$ mRNA, was detected. The results of the image analysis showed that the CHO cells transfected with the pEGFP-C1 plasmid expressed low levels of NGF $(0.15 \pm 0.02)$, and a weak (brightness) target band. The expression of $N G F$ mRNA in the CHO cells transfected with the pEGFP-C1-MAR-NGF plasmid was significantly higher $(0.25 \pm 0.03)$ than that in CHO cells transfected with the pEGFP-C1-NGF $(0.47$ $\pm 0.03)$ plasmid. NGF expression in both cell lines was significantly higher than that in normal $\mathrm{CHO}$ cells (Figure 2; $\mathrm{P}<0.05$ ).

\section{ELISA}

The pEGFP-C1-NGF and pEGFP-C1-MAR-NGF vectors were transformed into $\mathrm{CHO}$ cells. Stably expressing cell lines (selected using G418) were used to analyze the NGF 
concentration. NGF was detected in CHO cells transfected with the pEGFP-C1 plasmid. The NGF concentration was 1.93-fold higher in CHO cells transfected with the pEGFP-C1-MARNGF vector than in CHO cells expressing the pEGFP-C1-NGF vector. The NGF concentration was 1.22-fold higher in CHO cells expressing the pEGFP-C1-NGF compared to the cells expressing the pEGFP-C1 vector (Table 1).

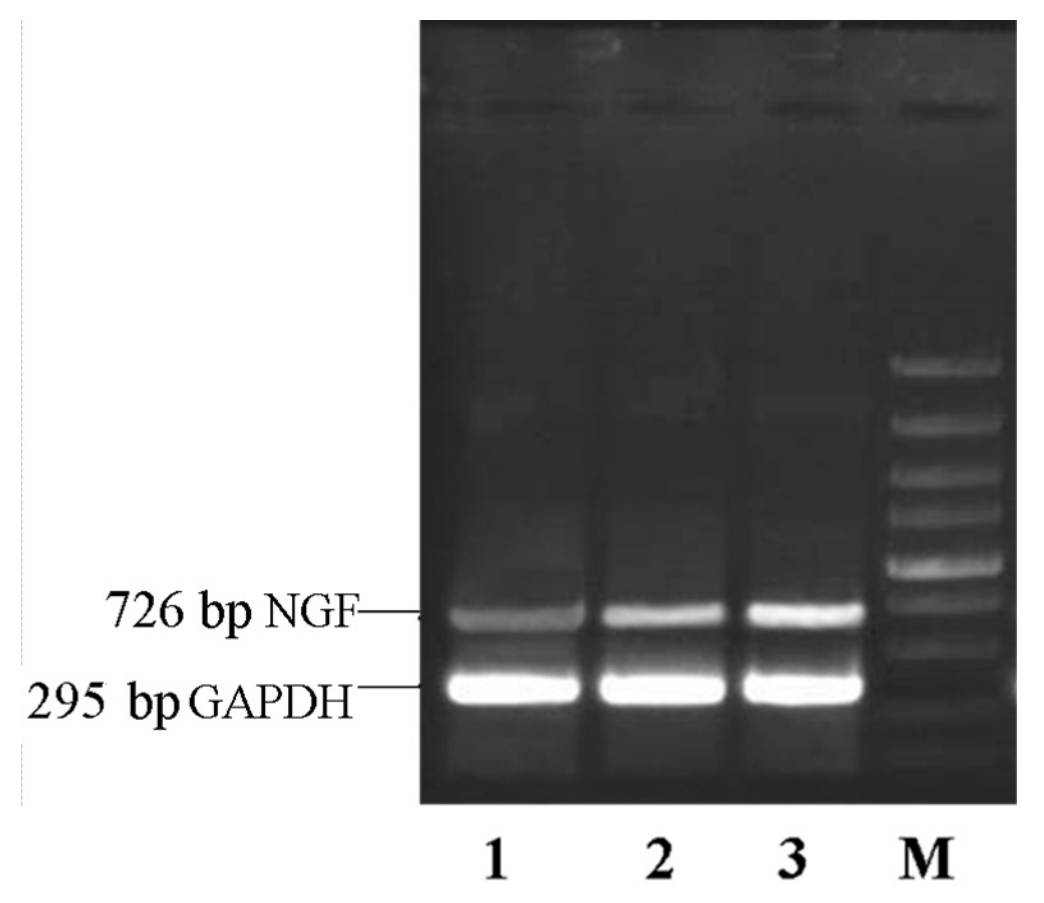

Figure 2. Expression of NGF mRNA was detected using RT-PCR. Lane $1=$ Normal CHO; lane 2 = pEGFP-C1NGF; lane 3 = pEGFP-C1-MAR-NGF; lane $M=$ DL 5000.

Table 1. NGF levels of CHO cells transfected with different vectors $\left(\mu \mathrm{g} \cdot \mathrm{L}^{-1} \cdot \mathrm{cell}^{-1}\right)$.

\begin{tabular}{lccc}
\hline & Normal CHO & pEGFP-C1-NGF & pEGFP-C1-MAR-NGF \\
\hline Sample (N) & 10 & 10 & 10 \\
Mean levels & $2.35 \times 10^{-6}$ & $2.87 \times 10^{-6}$ & $5.55 \times 10^{-6}$ \\
Fold ${ }^{1}$ & 1.22 & 1.93 \\
Fold $^{2}$ & & & \\
\hline${ }^{1}$ Represents pEGFP-C1-MAR-NGF $v s$ no transfect CHO. ${ }^{2}$ Represents pEGFP-C1-MAR-NGF $v s$ pEGFP-C1-NGF.
\end{tabular}

\section{Plasmid reduction}

The plasmids were not recovered from the CHO cells transfected with pEGFP-C1 and pEGFP-C1-NGF; however, the plasmids were retrieved from $\mathrm{CHO}$ cells expressing the pEGFP-C1-MAR-NGF vector; in addition, the digestion results were consistent with those of digested plasmids prior to transfection, using the KpnI/BamHI, HindIII/KpnI, and KpnI 
restriction enzymes. The MAR (367 bp) and NGF (726 bp) fragments were clearly detected (Figure 3).

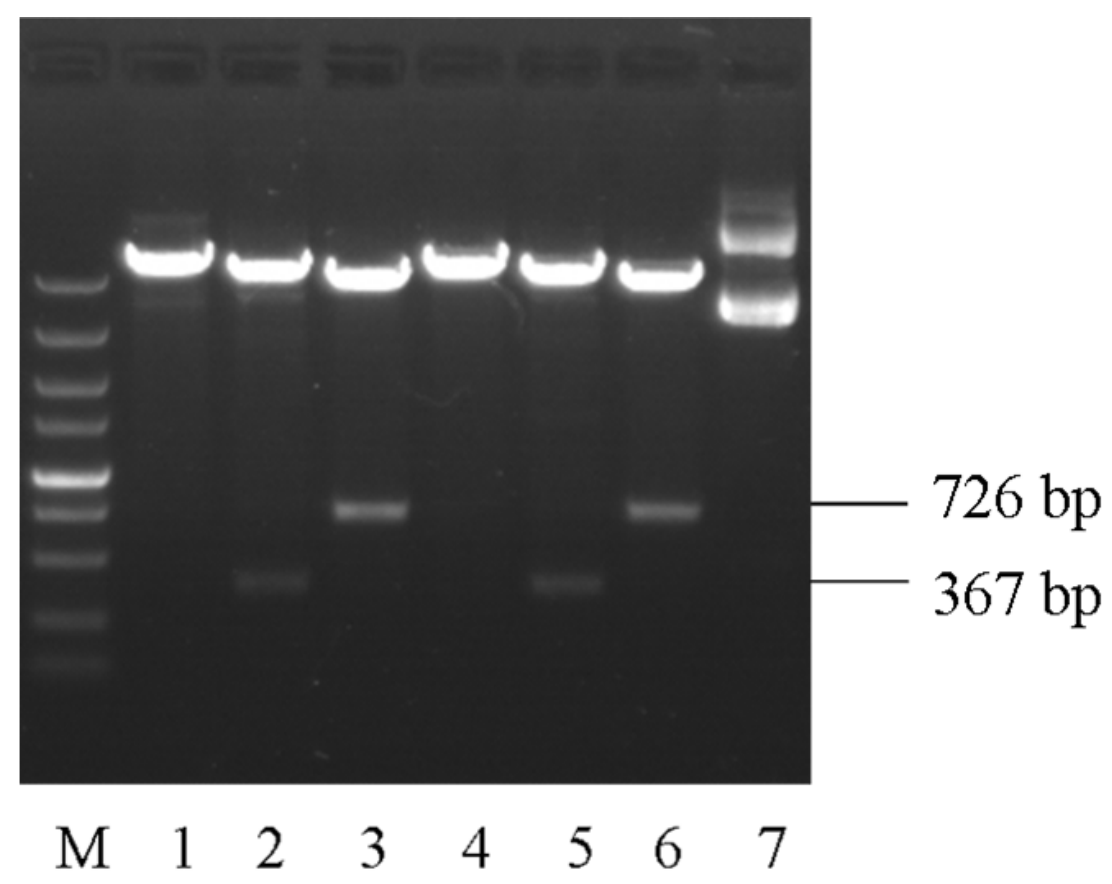

Figure 3. Enzyme digestion analysis of restored plasmids, transfected plasmids and plasmids before transfection. Lane $M=$ DL5000; lane 1 = restored plasmids in $\mathrm{CHO}$ cells are digested using KpnI; lane 2 = restored plasmids in $\mathrm{CHO}$ cells are doubly digested using $\mathrm{KpnI} / \mathrm{BamHI}$; lane $3=$ restored plasmids in CHO cells are doubly digested using Hind/KpnII; lane $4=$ transfected plasmids are digested using KpnI; lane $5=$ transfected plasmids are doubly digested using KpnI/BamHI; lane $6=$ transfected plasmids are doubly digested using Hind/KpnII; lane $7=$ Transfected plasmids.

\section{DISCUSSION}

Attached vectors can exist outside chromosomes in host cells to facilitate continuous and effective transgene expression. The use of attached vectors eliminates the potential risks of integrated vectors, such as the generation of mutations (Koirala et al., 2014). Attached vectors expressing the MAR element have attracted a lot of attention (Argyros et al., 2012; Mizutani et al., 2013; Verghese et al., 2014) because they are not integrated into the host (mammalian cell) genome. They bind to the matrix during the mitotic phase, and their stable attachment can prompt a highly effective, continuous, and safe expression. Simultaneously, these vectors exist with lower copy numbers in host cells, and replicate in the daughter cells based on the cell cycle. Previous studies have shown that a spliced DNA sequence (367 bp) from the MAR characteristic motif in human interferon- $\beta$ (2200 bp) can be used in the construction of recombinant vectors. It has been shown to mediate the attachment of vectors in mammalian cells with lower copy numbers, and enhance the 
expression of reporter genes (Lin et al., 2015). Attachment vectors with exogenous NGF genes mediated by MAR were constructed and transfected into $\mathrm{CHO}$ cells based on the results of preliminary studies. We aimed to identify if the MAR element could mediate the effective and safe insertion of a novel attached vector to mammalian cells, and the expression of the inserted exogenous target gene. This approach could provide a new tool for NGF gene therapy.

We observed low levels of NGF expression in CHO cells transfected with pEGFP-C1; on the other hand, the NGF concentration increased in CHO cells transfected with pEGFP-C1NGF. The NGF concentration was significantly increased in pEGFP-C1-MAR-NGF, compared to that of $\mathrm{CHO}$ cells transfected with pEGFP-C1-NGF. These results are in agreement with those of a previous study (Calado et al., 2014). RT-PCR analysis indicated a significantly increased target $N G F$ mRNA expression in CHO cells transfected with pEGFP-C1-MARNGF, compared to that in CHO cells transfected with the pEGFP-C1-NGF or pEGFP-C1 vectors. The MAR characteristic motif plays an important role in overcoming transgene silencing by affecting histone demethylation and acetylation; this in turn improves the transgene expression.

In this study, the target NGF gene was inserted into the multiple-cloning site of the pEGFP-C1 vector; the MAR sequence, on the other hand, was inserted downstream of NGF, in order to construct the novel attached vector. The plasmid reduction experiment indicated the stability and completeness (before and after transfection) of the MAR sequence, and the nonintegration of the recombinant plasmids into the genome (instead existing as attachments). These results were consistent with those of previous results (Hagedorn et al., 2011, 2012). Herein, we discovered that the MAR mediates vector attachment to the host cells and effectively increases the expression of the target $N G F$ gene. Cell culture experiments suggested that the NGF generated and secreted by astrocyte cells in vivo is temporal and transient, which results in a limited neuroprotective effect. Exogenous NGF is used to treat cerebral injuries in the laboratory and clinic. Therefore, novel attached vectors will provide a new tool for the clinical application of NGF.

Moreover, some studies have observed different expression levels of transgenes in different cells transfected with the MAR-attached vectors using different promoters (Tessadori et al., 2010; Haase et al., 2013). Therefore, further studies based on key regulatory elements in attached expression vectors, and on the suitability of host cells and mechanisms, are warranted. This approach could provide a theoretical and experimental basis for clinical applications of novel attached vectors in gene therapy.

\section{Conflicts of interest}

The authors declare no conflict of interest.

\section{ACKNOWLEDGMENTS}

Research supported by the National Natural Science Foundation of China (\#31300702) and the Scientific Research Fund of Xinxiang Medical University (\#2013ZD108). 


\section{REFERENCES}

Argyros O, Wong SP, Gowers K and Harbottle RP (2012). Genetic modification of cancer cells using non-viral, episomal S/MAR vectors for in vivo tumour modelling. PLoS One 7: e47920.

Buceta M, Galbete JL, Kostic C, Arsenijevic Y, et al. (2011). Use of human MAR elements to improve retroviral vector production. Gene Ther. 18: 7-13.

Calado SM, Oliveira AV, Machado S, Haase R, et al. (2014). Sustained gene expression in the retina by improved episomal vectors. Tissue Eng. Part A 20: 2692-2698.

Chiaretti A, Antonelli A, Genovese O, Fernandez E, et al. (2008) Intraventricular nerve growth factor infusion improves cerebral blood flow and stimulates double cortin expression in two infants with hypoxic-ischemic brain injury. Neurol. Res. 30: 223-228.

Ehrhardt A, Haase R, Schepers A, Deutsch MJ, et al. (2008). Episomal vectors for gene therapy. Curr. Gene Ther. 8: 147-161.

Girod PA, Nguyen DQ, Calabrese D, Puttini S, et al. (2007). Genome-wide prediction of matrix attachment regions that increase gene expression in mammalian cells. Nat. Methods 4: 747-753.

Haase R, Argyros O, Wong SP, Harbottle RP, et al. (2010) A significantly improved non-viral episomal expression vector for mammalian cells. BMC Biotechnol. 15: 20.

Haase R, Magnusson T, Su B, Kopp F, et al. (2013) Generation of a tumor- and tissue-specific episomal non-viral vector system. BMC Biotechnol. 13: 49.

Hagedorn C, Wong SP, Harbottle R and Lipps HJ (2011). Scaffold/matrix attached region-based nonviral episomal vectors. Hum. Gene Ther. 22: 915-923.

Hagedorn C, Baiker A, Postberg J, Ehrhardt A, et al. (2012). Rescue of S/MAR-containing nonviral episomal expression vectors. Cold Spring Harb. Protoc. 6: 709-711.

Harraghy N, Regamey A, Girod PA and Mermod N (2011). Identification of a potent MAR element from the mouse genome and assessment of its activity in stable and transient transfections. J. Biotechnol. 154: 11-20.

Harraghy N, Buceta M, Regamey A, Girod PA, et al. (2012). Using matrix attachment regions to improve recombinant protein production. Methods Mol. Biol. 801: 93-110.

Hirt B (1967). Selective extraction of polyoma DNA from in cell cultures. J. Mol. Biol. 26: 365-369.

Kamimura K, Suda T, Zhang G and Liu D (2011). Advance in gene delivery systems. Pharm. Med. 25: 293-306.

Koirala A, Conley SM and Naash MI (2014). Episomal maintenance of S/MAR-containing non-viral vectors for RPEbased diseases. Adv. Exp. Med. Biol. 801: 703-709.

Ley D, Harraghy N, Le Fourn V, Bire S, et al. (2013). MAR elements and transposons for improved transgene integration and expression. PLoS One 8: e62784.

Lin Y, Li Z, Wang T, Wang X, et al. (2015). MAR characteristic motifs mediate episomal vector in CHO cells. Gene 15: S0378-S1119.

Liu Y, Judd K and Lakshmipathy U (2013). Stable transfection using episomal vectors to create modified human embryonic stem cells. Methods Mol. Biol. 997: 263-272.

Mizutani A, Kikkawa E, Matsuno A, Shigenari A, et al. (2013). Modified S/MAR episomal vectors for stably expressing fluorescent protein-tagged transgenes with small cell-to-cell fluctuations. Anal. Biochem. 443: 113-116.

Mucller C and Flotte TR (2008). Clinical gene therapy using recombinant adeno-associated virus vectors. Gene Ther. 15: 858-863.

Tessadori F, Zeng K, Manders E, Riool M, et al. (2010). Stable S/MAR-based episomal vectors are regulated at the chromatin level. Chromosome Res. 18: 757-775.

Verghese SC, Goloviznina NA, Skinner AM, Lipps HJ, et al. (2014). S/MAR sequence confers long-term mitotic stability on non-integrating lentiviral vector episomes without selection. Nucleic Acids Res. 42: e53.

Zheng XR, Zhang SS, Yang YJ, Yin F, et al. (2010). Adenoviral vector-mediated transduction of VEGF improves neural functional recovery after hypoxia-ischemic brain damage in neonatal rats. Brain Res. Bull. 81: 372-377. 